\title{
Porous silicon solar cells
}

\author{
M. Balucani, K.Kholostov, V. Varlamava, F. Palma \\ Università di Roma La Sapienza, \\ Dipartimento di Ingegneria dell'Informazione, Elettronica e \\ Telecomunicazioni, \\ via Eudossiana 18, 00184 Roma, Italy
}

\author{
M. Izzi, L. Serenelli, M. Tucci \\ ENEA Research Centre Casaccia \\ Via Anguillarese, 301, 00123 Roma, Italy
}

\begin{abstract}
We developed a new process for the fabrication of crystalline solar cell, based on an ultrathin silicon membrane, taking advantage of porous silicon technology. The suggested architecture allows the costs reduction of silicon based solar cell reusing the same wafer to produce a great number of membranes. The architectures combines the efficiency of crystalline silicon solar cell, with the great absorption of porous silicon, and with a more efficient way to use the material. The new process faces the main challenge to achieve an effective and not expensive passivation of the porous silicon surface, in order to achieve an efficient photovoltaic device. At the same time the process suggests a smart way to selective doping of the macroporous silicon layers despite the through-going pores.
\end{abstract}

Keywords—silicon nanoelectronics, nanofabrication ,porous silicon, solar cells.

\section{INTRODUCTION}

The decrease of silicon consumption through the wafer thickness reduction together with low surface reflection is one of the current research challenge in crystalline silicon photovoltaics [1]. The use of macroporous silicon is one of several options to fabricate thin crystalline silicon absorber layers with very low reflection [2], [3]. With this approach, the macroporous silicon layer can be directly used as thin-film absorber in solar cell. Indeed the macroporous structure guarantees a low surface reflectance and an efficient light trapping. Nevertheless it is extremely challenging the possibility to perform an effective and not expensive passivation by surface selective doping of the porous surface despite the through-going pores, in order to achieve an efficient photovoltaic behavior.

In literature are reported different types of junction formation using macroporous silicon as the absorber layer of solar cell [4], [5]. Heterojunction device process was initially adopted, achieving an energy-conversion efficiency of $7.2 \%$ [4]. Other approaches used the growth of a $\mathrm{p}^{+}$-type layer by epitaxial growth onto n-type macroporous silicon layer as junction formation [5]. In this case the epitaxial layer covers the full outer and inner surface of the macroporous layer thus improving the energy-conversion efficiency up to $13.1 \%$ with a remarkable high short-circuit current density of $37.1 \mathrm{~mA} / \mathrm{cm}^{2}$ on a $35 \mu \mathrm{m}$ thick macroporous silicon solar cell.

Recently the use ion implantation to selectively dope the outer surfaces of the macroporous silicon layer has been proposed [6]. The implantation depth inside the pores was controlled by the incident angle of implantation, avoiding complex steps for side-selective doping.

We are developing a new process to fabricate crystalline solar cell based on ultrathin silicon membrane, taking advantage of the technology of porous silicon.

The device architecture consists of a macro-porous membrane, having a deeply textured surface capable, to efficiently capture the impinging light. Then the light is absorbed in the porous structures, and the photo-generated carriers are delivering to contacts deposited at the edges of the ultra-thin semiconductor layer.

The new fabrication method of the ultra-thin silicon layer cut from a silicon wafer, is based on the possibility to create macroporous structures in the silicon, by using the proper anodization regime, and detach this structure from the substrate through formation and subsequent etching of a layer of nanoporous silicon underneath it, obtaining very thin membrane as a result.

The anodization process performed with the presence of the $\mathrm{n}$ doped layer has produced a peculiar geometrical structure, which allowed a dome-like shape of the pores beside the junction. This structure permits an efficient formation of the contact on the n-emitter by ITO deposited by sputtering.

The effective decrease of the density of surface electronic defects, after passivation, was tested by the measurement of carrier lifetime, achieved by a Sinton test setup

The contact on the p-type side was obtained by aluminum deposition and subsequent local laser treatment.

The overall cell structure, formed as a membrane, has a thickness of $18 \mu \mathrm{m}$. The membrane was deposited and fixed on glass covered with ITO.

Then the cell was measured at room temperature in dark and under AM1.5G sun light conditions.

At the moment the rectifying behavior of the proposed junction appears not jet optimized. Nevertheless the cell shows a large current collection under sunlight exposure as evidence of excellent scattering process induced by the porous structure and surface passivation.

The work was carried out under EU Program ERG. 


\section{Cell Fabrication}

\section{A. The solar cell structure}

Fig.1 reports the structure of the ultrathin porous silicon membrane solar cell. A n-p junction was formed on the front face of the membrane, before the pores formation. The junction was maintained during the pore formation and remains active at the edges of the separation baffles on the pores.

An ITO layer deposition ensures the ohmic contact to the n-type layer. Once detached from the silicon wafer, the membrane was deposited onto a glass substrate, on which a transparent ITO layer has been formerly deposited as contact. The mechanical contact between this layer and the ITO layer deposited on the porous silicon membrane ensures the current collection.

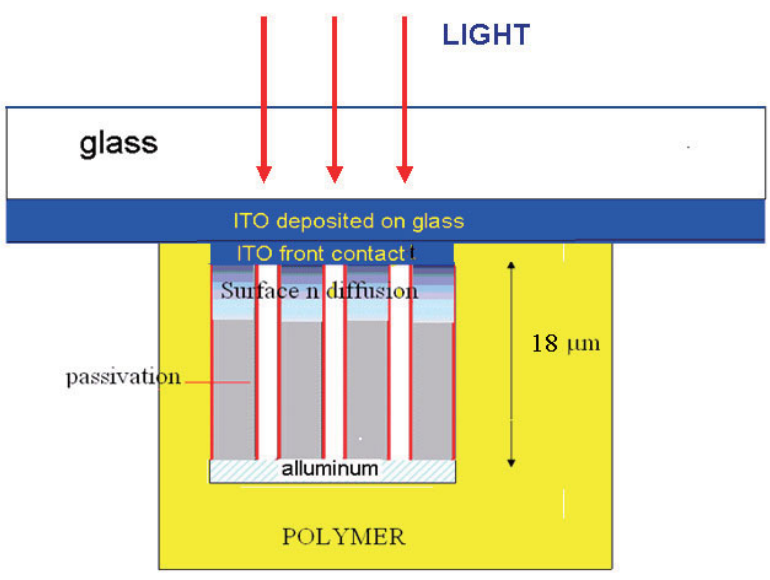

Fig. 1. The solar cell structure as obtained from the porous silicon membrane.

\section{B. The membrane formation}

In the proposed process, a $4 \mu \mathrm{m}$ thick macroporous silicon layer was first formed using anodization in galvanostatic regime in p-type $<100>$ crystalline silicon substrates with 10 $20 \Omega \cdot \mathrm{cm}$ bulk resistivity [7]. The anodization electrolyte was composed of hydrofluoric acid (50 wt.\%) and dimethyl sulfoxide mixed in 10:50 ratio. The anodization current density was $8 \mathrm{~mA} / \mathrm{cm}^{2}$.

We chose to create a standard emitter junction, before the final pore etching. Standard emitter $60 \Omega / \mathrm{sq}$ p-n junction was promoted by diffusion of phosphorus over the obtained macroporous structure, on the $p$ type silicon wafer by phosphorous doping in open furnace tube. The depth of the junction was around $0.36 \mu \mathrm{m}$. On this formerly diffused $n-p$ junction, we perform a second porous silicon etching. to grow the thickness of the macroporous structure up to $14 \mu \mathrm{m}$ using the same anodization regime.

$\mathrm{N}$-type doped silicon appears to be more resistant to the electrochemical etching with respect to p-type doped silicon.
Indeed the etching process produces only small holes on the ntype layer. Instead, continuing the etching on p-type silicon results in regular size pores. As shown in Fig.1, a thin silicon layer was formed due to the presence of the n-type doped emitter layer at the edge of each porous, with a vaulted shape. This thin silicon layer, connecting the porous holes, increases the membrane mechanical rigidity and favors the deposition of the contact layer.

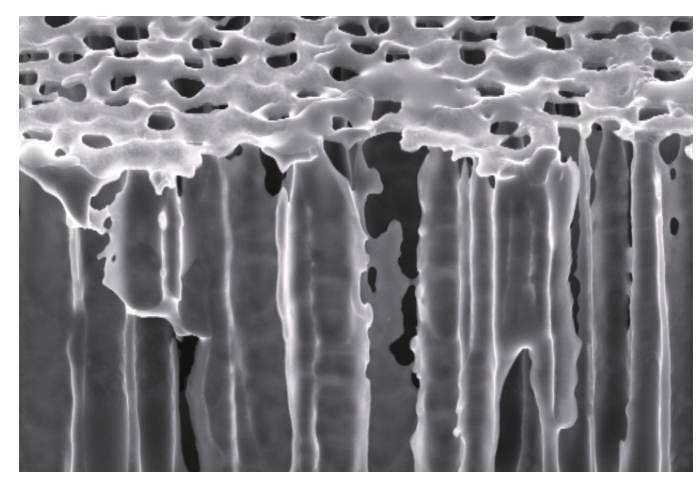

Fig. 2. SEM picture of the porous structure, with reduced top aperture due to the n-type doped emitter at the top of each holes.

The new approach presented in this paper tries, as first step, to answer to the requirement of selective doping of the macroporous Si layers despite the through-going pores. We noticed that the formation of a doped layer "after" the pore creation, and after the membrane dethatched from the wafer, introduces a relevant complexity in the device fabrication process. High temperature processes, as epitaxial growth, or vacuum processes, as needed for ion implantation, may represents critical steps. In particular the handling of the fragile membranes may dramatically reduce any process yield.

Subsequent nanoporous etching permits the creation of a chamber underneath the porous layer, large enough to create a continuous cut zone underneath the macroporous layer useful to form the membrane, as shown in Fig.2. The membranes has a thicknesses ranging from 10 to $30 \mu \mathrm{m}$.

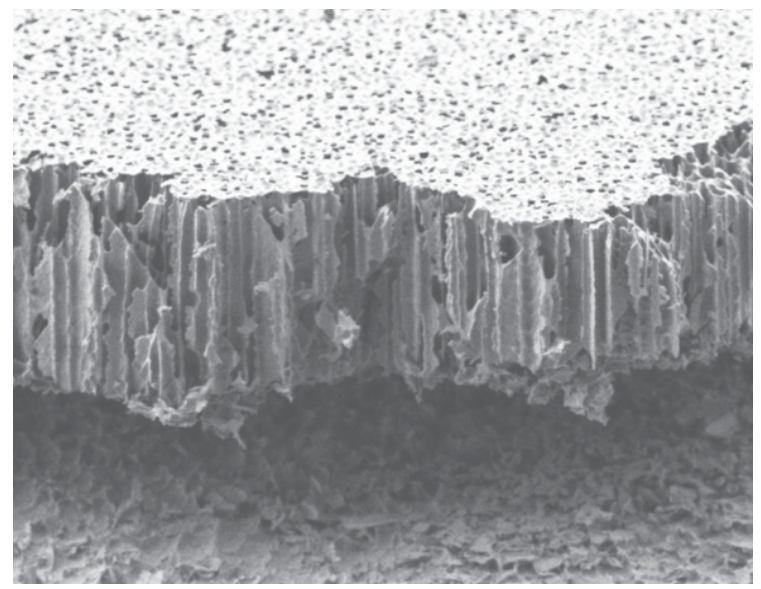


Fig. 3. SEM picture of the porous silicon membrane.

\section{Passivation}

Before dethatching the membrane, a passivation process was applied to the porous structure, included the n-p junction previously formed on the silicon surface. The process consists of an additional very light n-type doping.

Recombination lifetime was measured by Sinton WT100 system to evaluate the photoconductivity decay of minority carrier lifetime of the entire wafer. While before the passivation the lifetime was around 1-3 $\mu$ sec, after the passivation with the light doping, the measurement on porous silicon formed directly on p-type wafer, gives a lifetime of 120 $\mu$ sec. This value allows sufficient collection of photo-generated carrier in the very thin membrane. Whereas the lifetime measurement on the porous silicon after the n-type emitter formation on the p-type wafer shows a value of $50 \mu \mathrm{sec}$, that still it is a relevant value. The lifetime reduction can be attributed to additional recombination due to the n-type doped region.

A microwave method for the characterization of the lifetime in the first few micron from the porous surface, recently developed [8], confirmed that the reduction of lifetime actually occurs at the porous surface.

\section{Contacts}

Beside the $\mathrm{n}$ layer, the structure presents only narrow holes, thus it furnishes higher mechanical stiffness. A deposition of ITO permits the formation of both a contact and an antireflection coating [9]. The increased surface, due to the narrow holes, and the small dimension of the apertures prevents the penetration of ITO film inside the porous during the sputtering process at $350^{\circ} \mathrm{C}$ by sputtering. Fig. 4 shows a SEM picture of ITO film over the emitter.

After mechanical detachment, the membrane was attached on an ITO covered glass substrate. Then the two ITO layers are now in tight contact.

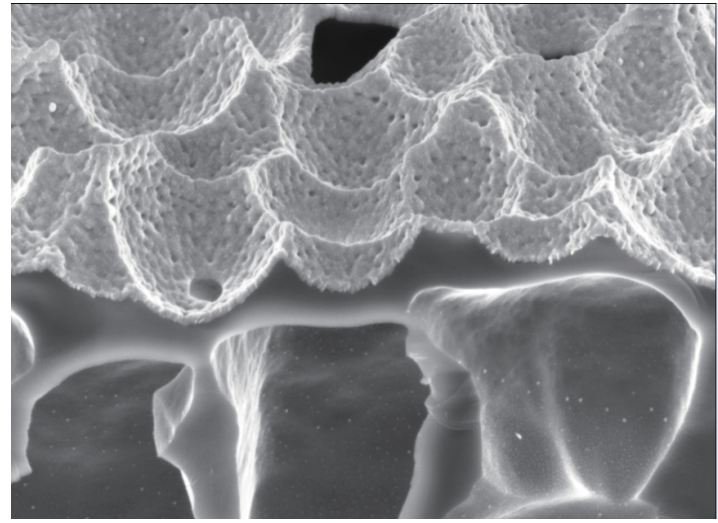

Fig. 4. SEM picture of the ITO contact on the $n$ doped surface.

The ohmic contact on the low doped p-type silicon wafer represents the back contact. It was obtained by activation under laser local treatment of $300 \mathrm{~nm}$ aluminum, deposited by grazing evaporation. A study of the laser activation intensity and focus has permitted to obtain partial melting of silicon at the surface reduce, as shown in Fig. 5, and to substantially reduce the contact resistivity.

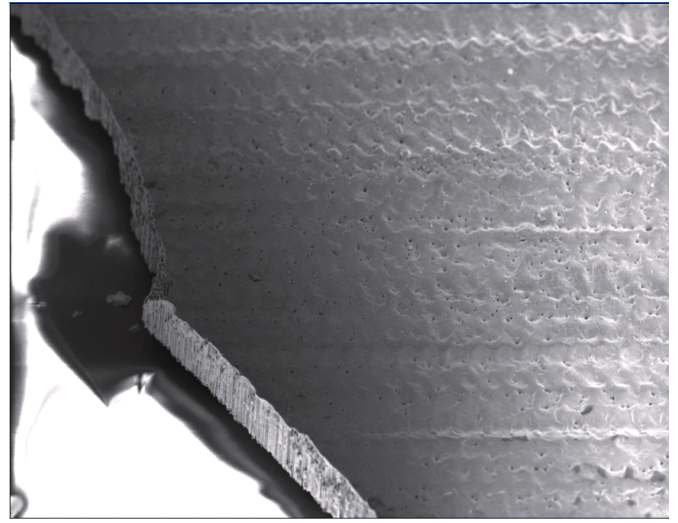

Fig. 5. SEM picture of the alluminum contact on the p doped surface of the membrane.

\section{CELl CHARACTERIZATION}

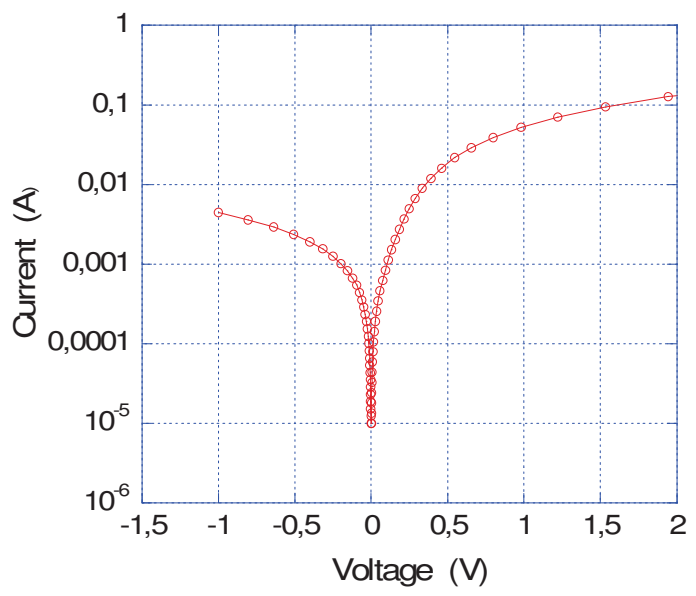

Fig. 6. I-V measurement of the cell in dark condition.

Standard I-V measurements were performed on the cell to evaluate the rectifying behavior of the junction as well as the photovoltaic performances under sunlight simulator in AM1.5G conditions. I-V in dark measurements shows that the rectifying junction appears even if not yet optimized. This characteristic is shown in Fig.6. Nevertheless, the new device architecture of ultrathin solar cell shows a high current collection under AM1.5 illumination as reported in Fig.7. This large value is mainly due to the excellent absorption process induced by the porous structure, and to the good passivation achieved. 


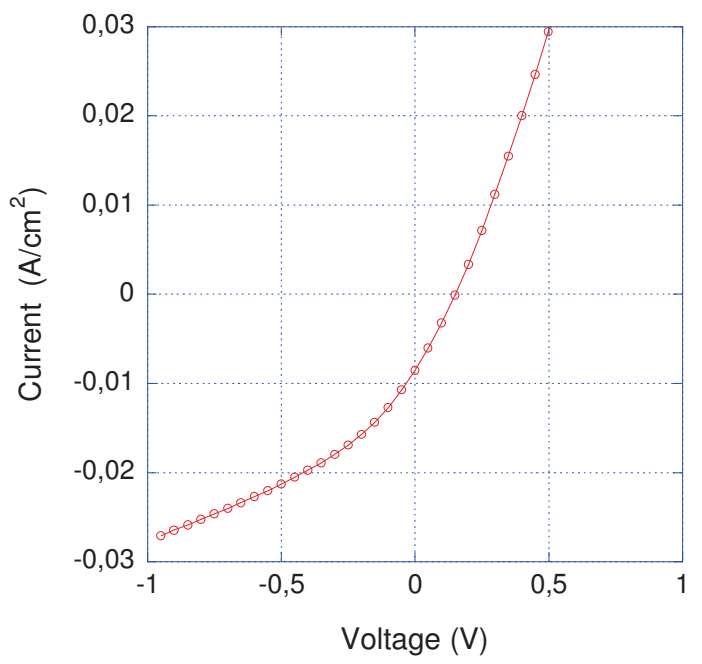

Fig. 7. I-V measurement under sun simulator in AM1.5G condition.

\section{CONCLUSIONS}

We are developing an innovative process to fabricate of a novel device architecture of crystalline solar cell, based on ultrathin silicon membrane.

The device architecture consists of a macro-porous membrane that offers a deeply textured surface capable to efficiently capture the impinging light as absorbed it in the porous structure.

The suggested process addresses the two main challenges of this structure, i) to achieve an effective and not expensive passivation of the porous surface, in order to ensure an efficient photovoltaic behavior; ii) to achieve a selective doping of the macroporous Si layers despite the through-going pores.
Up to now the p-n junction embedded in the porous membrane is not yet optimized, nevertheless the suggested device architecture of ultrathin solar cell shows a large value of current collection under AM1.5 illumination up to 25 $\mathrm{mA} / \mathrm{cm}^{2}$, mainly due to the excellent absorption process induced by the porous structure, and the good passivation achieved, demonstrating the effectiveness of the new process of fabrication of the ultrathin architecture.

\section{ACKNOWLEDGMENT}

Authors whish to thank Sapienza Nanotechnology and Nanoscience Center for the access to the SEM facility.

\section{REFERENCES}

[1] ITRPV 2012, International Technology Roadmap for Photovoltaics Results 2012, Third Edition, Berlin 2012, www.ITRPV.net.

[2] Lehmann V, Hönlein W, Stengl R, Willer J, Wendt H. Verfahren zur Herstellung einer Solarzelle aus einer Substratscheibe, German patent DE4204455C1; Filing date: 29.01.1992.

[3] Brendel R, Ernst M., Macroporous Si as an absorber for thin-film solar cell,. Phys. Status Solidi RRL 2010, pp.40-42.

[4] Ernst M, Brendel R, Ferré R, Harder N.,Thin macroporous silicon heterojunction solar cells, Phys. Status Solidi RRL, 2012, pp. 187-189.

[5] Ernst M, Brendel R., Macroporous Silicon Solar Cells With an Epitaxial Emitter, IEEE J. Photovoltaics, 2013, pp. 723 - 729.

[6] Marco Ernst, Henning Schulte-Huxel, Raphael Niepelt, Sarah KajariSchröder, Rolf Brende, Thin crystalline macroporous silicon solar cells with ion implanted emitter, Energy Procedia 38, 2013 , pp.910-918.

[7] Nenzi P., Kholostov K., Crescenzi R., Bondarenka H., Bondarenko V., Balucani M., Electrochemically etched TSV for porous silicon interposer technologies, Electronic Components and Technology Conference (ECTC), 2013 IEEE 63rd, 2201-2205.

[8] Perticaroli S., Varlamava V., Palma F., Microwave sensing of nanostructured semiconductor surfaces, Applied Physics Letters 104, 2014, 013110

[9] de Cesare M., Caputo D., Tucci M., Electrical Properties of ITO/Crystalline-Silicon, Contact at Different Deposition Temperatures, IEEE Electron Device Letters, VOL. 33, NO. 3, 2012, pp. 327-329 